\title{
THE RELATIONSHIP BETWEEN PUPIL DIAMETER AND DECENTRATION IN MYOPIA
}

\author{
R. NUZZI, C. FINAZZO and L. FRANCONE \\ Turin, Italy
}

\section{SUMMARY}

Since optical zone centration is of such importance in refractive surgery, we have studied, with a computeraided videopupillograph, the problem of identifying it in myopes. The findings of pupil centre shift as a function of pupil diameter and degree of myopia are reported in two groups of myopes (low and medium) and a control group. Results indicate that the amount and direction of decentration vary according to pupil diameter and degree of myopia. No generally valid instructions can be laid down and each case must be treated on its merits.

Optical zone centration is critical in refractive surgical procedures, and inappropriate centring can cause glare and ghost images in the dark when the pupil remains in mydriasis for a long time. ${ }^{1-7}$ The optimal centration point is debatable: should it be pupil centre entrance, the geometric corneal centre for the optical zone $\mathrm{e}^{3}$ or the point of intersection of the visual axis with the cornea? If the pupillary centre is chosen, finding it may not be so easy, since the pupil does not dilate concentrically and its centre moves as its diameter varies. ${ }^{3,8}$ This shift is superonasal in miosis and no relationship is to be found between it and degree of mydriasis. ${ }^{2,9}$ Optic zone localisation may also vary with respect to the points used for centring depending on luminance and pupil diameter. Pupil centre shift from the visual axis may be temporal by up to $0.75 \mathrm{~mm}$ and from the corneal centre by up to $1.06 \mathrm{~mm} .{ }^{10}$

The present study quantifies pupil centre shift topographically from the corneal geometric centre according to degree of myopia and pupil diameter.

From: Institute of Ophthalmology, University of Turin, Turin, Italy.

Correspondence to: Dr R. Nuzzi, Istituto di Clinica Oculistica, Via Juvarra 19, I-10122 Turin, Italy. Tel: (+3911) 56.29.047. Fax: (+3911) 53.90.24.

\section{MATERIALS AND METHODS}

The study was carried out with an infrared computerised videopupillograph (Hamamatsu C301 Iriscorder; Fig. 1) on a randomised sample of 41 myopic subjects ( 82 eyes) aged between 18 and 39 years (mean 26.21 years, SD 4.2 years). The sample was divided into two groups: low myopia (spherical equivalent refraction $-0.25 \mathrm{D}$ to $-3.50 \mathrm{D})$ with normal axial length (22-24 $\mathrm{mm}$ ) and medium myopia (spherical equivalent refraction $-3.75 \mathrm{D}$ to $-8.00 \mathrm{D}$ ) with elongated axial length $(24-30 \mathrm{~mm})$. In the low myopia group there were 17 subjects $(11$ men, 6 women) and in the medium myopia group 24 subjects (22 men, 2 women).

A control group was formed of 21 emmetropic subjects ( 12 women, 9 men), aged between 18 and 37 years, whose mean age (25.2 years, SD 5.2 years) corresponded to that of the study sample.

Exclusion criteria included: ophthalmic pathologies of the adnexa, anterior segment and posterior segment; crystalline lens transmittance alterations; major myopic-type retinal changes; amblyopia; and drug therapy capable of affecting pupil statics and/or dynamics.

Each subject was given a complete eye examination including a cycloplegic refraction examination

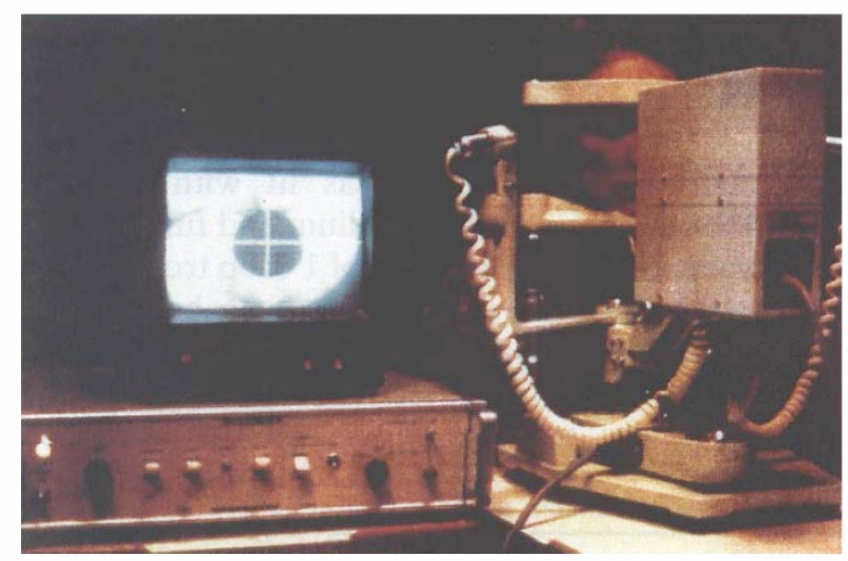

Fig. 1. Hamamatsu C301 Iriscorder videopupillograph. 


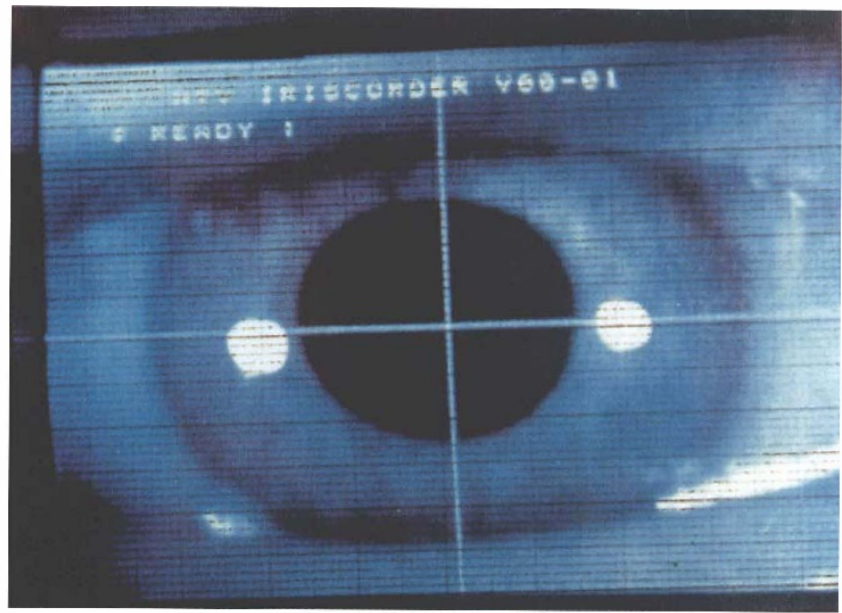

Fig. 2. Hamamatsu C301 Iriscorder videopupillograph television system for pupil image viewing.

with retinoscopy and autorefractometry; the amount of astigmatism was no more than $1.50 \mathrm{D}$.

After receiving informed consent from the patient, correct pupil centre and corneal geometric centre were localised by videopupillographic examination, including on the video a sheet of millimetre-squared tracing paper on which, before the pupillographic recordings in the different conditions (basal, miosis, 15 and 25 minutes of mydriasis) were made, the corneal geometric centre was marked. This was taken to be the point of intersection of the vertical and horizontal meridians with the iris periphery considered as the corneal circumference.

A $100 \mu \mathrm{m}^{2}$ calibrated screen was placed on the telecamera objective. First, a millimetre rule was placed before each eye and the corneal horizontal and vertical diameters found. These measurements were useful in the pupil decentration computing phase as comparative and confirmatory values of the corneal diameters extracted from the video images with the squared paper after scale adjustment.

Each eye's pupillographic data and pupil centre shift from the corneal geometric centre were recorded under four different pupil conditions: basal (in the dark after 15 minutes adaptation time, as for the conventional pupillographic examinations); in miosis (under better lighting conditions in which the room of about $12 \mathrm{~m}^{2}$ was lit with a $60 \mathrm{~W}$ incandescent lamp); and in medium and full mydriasis (obtained by the instillation of 1 drop tropicamide $1 \%$ eyewash in both eyes, measurements being made at 15 and 25 minutes after instillation).

Each eye's pupil centre under each condition was marked on the squared paper and photographed with a Yashika F-1000 camera on ASA 200 film (Fig. 2). The limbus was traced, as was the border of the pupil. The limbal centre and the centre of each of the four pupil perimeters were calculated and their locations marked by computer using morphometric analysis. ${ }^{11}$ The geometric centre of the cornea defined by the centre of the limbal trace was defined as the origin of a Cartesian coordinate system.

We have investigated the geometric centre of the pupil for the following reasons. In clinical practice surgeons should centre the optical zone on the coaxially sighted corneal reflex. We know that the visual axis does not coincide with the entrance pupil centre and the coaxially sighted corneal reflex is the visual axis corneal intercept found by the surgeon with the coaxial microscope light. Refractive surgery procedures modify the corneal area overhanging the pupil, but the light bundle intercepting the central corneal area and entering the eye shifts as a result of pupil dynamics. Therefore this study investigated whether it is possible to state the relationship between pupil size and entrance pupil centre by locating the centre of the pupil.

To find the amount of pupil centre shift with respect to the corneal geometric centre for each eye, we: (1) calculated pupillary distance from the corneal geometric centre as shown on the squared paper vectorially for each individual pupil condition and (2) translated the data into real terms by means of the scale, suitable for minimum distance measurements of $0.125 \mathrm{~mm}$, given by the ratio between the real pupil diameter sizes obtained by pupillography and the photographed sizes.

All the data were analysed mathematically and statistically. Statistical comparisons were performed using an analysis of variance (ANOVA).

\section{RESULTS}

\section{Pupil Diameter, D1 (Table I)}

In low myopia, D1 was significantly lower $(p<0.001)$ than in emmetropic controls in basal conditions but significantly higher $(p<0.001)$ in miosis. In mydriasis at 15 minutes there was no significant difference relative to the emmetropic controls, while at 25 minutes D1 was appreciably lower than that of the emmetropes $(p<0.01)$.

In medium myopia there was no significant difference in D1 relative to the controls.

Table I. Pupil diameter: mean values (mm)

\begin{tabular}{lcccc}
\hline & Miosis & Basal & Mydriasis at 15 min & Mydriasis at 25 min \\
\hline Emmetropes & $3.26 \pm 0.20$ & $5.72 \pm 0.16$ & $6.49 \pm 1.70$ & $7.71 \pm 0.73$ \\
Low myopes & $3.68 \pm 0.39$ & $5.16 \pm 0.27$ & $6.84 \pm 0.13$ & $7.17 \pm 0.12$ \\
Medium myopes & $3.52 \pm 0.66$ & $5.92 \pm 0.59$ & $6.79 \pm 0.64$ & $7.38 \pm 0.97$ \\
\hline
\end{tabular}


Table II. Distance between corneal geometric centre and pupil centre ( $\mathrm{mm}$ )

\begin{tabular}{lcccc}
\hline & Miosis & Basal & Mydriasis at 15 min & Mydriasis at 25 min \\
\hline Emmetropes & $0.43 \pm 0.09$ & $0.38 \pm 0.05$ & $0.58 \pm 0.07$ & $0.64 \pm 0.08$ \\
Low myopes & $0.57 \pm 0.08$ & $0.28 \pm 0.05$ & $0.53 \pm 0.07$ & $0.61 \pm 0.04$ \\
Medium myopes & $0.61 \pm 0.08$ & $0.34 \pm 0.04$ & $0.72 \pm 0.05$ & $0.69 \pm 0.07$ \\
\hline
\end{tabular}

\section{Pupil Decentration Data (Table II)}

A statistical interference test for non-paired data was used because the two myope subgroups and the control group were of different sizes.

In emmetropes the maximum shift was $0.26 \mathrm{~mm}$ (at 25 minutes mydriasis); the change in pupil size associated with this shift was $1.99 \mathrm{~mm}$.

In low myopia there was less pupil centre shift in basal conditions $(p<0.01)$, but more in miosis $(p<0.001)$, than in the controls; the mean shift at 15 minutes mydriasis was less than that of the controls $(p<0.05)$. Maximum shift was 0.33 (at 25 minutes mydriasis), associated with a $2.01 \mathrm{~mm}$ change in pupil size.

In medium myopia, in basal conditions, the shift was less than in the emmetropes but not so much as that of the low myopes $(p<0.01)$. In miosis, the shift was greater than in the controls. In mydriasis the mean shift at 15 minutes was maintained at 25 minutes, its value being higher in the myopes $(p<0.05)$. The maximum shift in the medium myopes was $0.38 \mathrm{~mm}$ (at 15 minutes mydriasis), associated with a $0.87 \mathrm{~mm}$ change in pupil size.

\section{Shift Direction Data Analysis: Pupil Centre vs Corneal Geometric Centre (Table III)}

In emmetropes, in basal conditions, most pupil centres were in the superior temporal sector. In miosis, decentration was overwhelmingly superonasally. In mydriasis the mean shift at 15 minutes was maintained at 25 minutes, its value being higher in the myopes.

In low myopia, in basal conditions, there was no decentration. In miosis, more than half the subjects showed supero-temporal decentration and more than one third a supero-nasal shift. In mydriasis, the direction of shift was overwhelmingly superotemporal.

In medium myopia, basal superior nasal sector localisation was not affected by miosis. In mydriasis, decentration at 15 minutes was into the superior temporal sector and at 25 minutes in the superotemporal direction.

\section{DISCUSSION}

All corneal refractive surgery techniques are based on modification of the refractive power of that part of the cornea through which light rays pass on their way to the fovea. The efficacy of the surgery and/or post-operative complications depend largely on the accuracy with which the surgeon identifies the optic zone and the point on which it centres. ${ }^{12-14}$ There may result monocular diplopia from a decentred optic zone,${ }^{15}$ image distortion in radial keratotomy, and visual axis decentration in keratomileusis or epikeratophakia. ${ }^{2-4.16}$ Glare after radial keratotomy may be due to distortion of light transmission through the corneal zones where the incisions partly cover the pupillary area. ${ }^{7}$ Therefore, the central clear optical zone should be made to coincide with the pupil centre instead of with the visual axis, though this is still a bone of contention. ${ }^{10,17,18}$

The maximum tolerable decentration to avoid visual disturbances in surgical or parasurgical refractive corrections ranges from 0.5 to $1 \mathrm{~mm}$ according to the school of thought. ${ }^{3,7.17}$ Theoretically, the optimal centration point to use is the coaxially sighted corneal reflex. The entrance pupil centre is, in

Table III. Direction of pupil centre shift

\begin{tabular}{|c|c|c|c|c|c|}
\hline & Indifferent & Supero-nasal & Supero-temporal & Infero-nasal & Infero-temporal \\
\hline $\begin{array}{l}\text { Miosis } \\
\text { E } \\
\text { LM } \\
\text { MM }\end{array}$ & 9.52 & $\begin{array}{l}90.48 \\
35.30 \\
95.84\end{array}$ & $\begin{array}{r}58.82 \\
4.16\end{array}$ & 5.88 & \\
\hline $\begin{array}{l}\text { Basal } \\
\text { E } \\
\text { LM } \\
\text { MM }\end{array}$ & $\begin{array}{r}4.76 \\
100.00\end{array}$ & $\begin{array}{l}23.81 \\
91.68\end{array}$ & $\begin{array}{r}71.43 \\
4.16\end{array}$ & 4.16 & \\
\hline $\begin{array}{l}\text { Mydriasis at } 15 \mathrm{~min} \\
\text { E } \\
\text { LM } \\
\text { MM }\end{array}$ & 9.52 & & $\begin{array}{r}90.48 \\
100.00 \\
95.84\end{array}$ & & \\
\hline $\begin{array}{l}\text { Mydriasis at } 25 \mathrm{~min} \\
\text { E } \\
\text { LM } \\
\text { MM }\end{array}$ & & 14.28 & $\begin{array}{r}85.72 \\
94.12 \\
100.00\end{array}$ & 5.88 & \\
\hline
\end{tabular}

E, emmetropes; LM, low myopes; MM, medium myopes. 
theory, a well-defined point, but in practice its position varies with pupil size, ambient lighting and the pupil's eccentric progressive dilation. ${ }^{19-22}$ Its shift can be as much as $0.5 \mathrm{~mm}$ in mydriasis with unpredictable extent and direction. ${ }^{3,8}$ The shift is supero-nasal in miosis, but there is no sure correlation between the extent of the shift and the degree of mydriasis. ${ }^{2,9}$

We found that: (1) in basal conditions, myopic pupil decentration is less than that in emmetropes; (2) miotic myopic decentration increases; (3) at 15 minutes and 25 minutes mydriatic shift is slightly less in medium than in low myopes, but markedly higher than in emmetropes. There is, therefore, a significant pupil centre shift in the different pupil diameter conditions.

This may be of importance since some visual disturbances typical of subjects after radial keratotomy occur in low ambient lighting when the pupil is moderately mydriatic. Decentration in basal conditions is supero-temporal in emmetropes, absent in low myopes and mainly supero-nasal in medium myopes. In miosis, decentration in emmetropes and medium myopes is mainly into the superior nasal sector, but in low myopes movement is $2: 1$ superior temporal sector to superior nasal sector. At 15 minutes and 25 minutes mydriasis, decentration is uniform in all three groups and is into the superior temporal sector.

In conclusion, suitably locating the optic zone in surgery to avoid post-operative complications requires taking into account that the pupil shift from the corneal geometric centre may be as much as $0.72 \mathrm{~mm}$ and that its direction may vary depending on pupil diameter and refractive state.

Key words: Pupillography, Myopia, Pupil diameter, Pupil shift.

\section{REFERENCES}

1. Borgmann $\mathrm{H}$. The behaviour of the pupillary diameter in darkness after preliminary illumination of different duration. Graefes Arch Ophthalmol 1967;172:220-9.

2. Walsh PM, Guyton PL. Comparison of two methods of marking the visual axis on the cornea during radial keratotomy [correspondence]. Am J Ophthalmol 1984; 97:660-73.

3. Uozato H, Guyton LD. Centering corneal surgical procedures. Am J Ophthalmol 1987;103:264-75.

4. Merlin U. Appunti di tecnica per la correzione delle miopie lievi e medie e dell'astigmatismo congenito mediante cheratotomie non perforanti. Atti del Corso di Chirurgia Refr., Sior. Milan:1991:1.
5. McDonald MB, Liu JC, Byrd TJ, Abdelmegeed M, Angotti Andrade H, Klyce SD, Varnell L, Munnerlyn CR, Clapham TN, Kaufman HE. Central photorefractive keratectomy for myopia. Ophthalmology 1991; 98:1327-41.

6. Nuzzi R, Americo G. Pupillographic investigation in myopia. New Trends Ophthalmol 1992;7:311-9.

7. Doane JF, Cavanaugh TB. Optical zone centration for keratorefractory surgery. Ophthalmology 1994;101: 215-26.

8. Wilson SE, Klyce SD. Quantitative description of corneal topography. Arch Ophthalmol 1991;109: 349-62.

9. Fay AM, Trokel SL, Myers JA. Pupil diameter and the principal ray. J Cataract Refract Surg 1992;18:348-56.

10. Pande M, Hillman JS. Optical zone centration in keratorefractive surgery: entrance pupil centre, visual axis, coaxially sighted corneal reflex or geometric corneal center? Ophthalmology 1993;100:1230-42.

11. Panzica F, Viglietti E, D'Ambrosio C, Haggi G. Un nuovo programma morfometrico computerizzato. Stat Med 1987;43:503-10.

12. Aron-Rosa DS, Boerner CF, Bath P, Gross M, Tomsit JC, True L, Hufnagel T. Corneal wound healing after excimer laser keratotomy in a human eye. Am J Ophthalmol 1987;103:454-60.

13. Maloney RK. Corneal topography and optical zone location in photorefractive keratectomy. J Refract Corneal Surg 1990;6:363-75.

14. Marshall J, Trokel SL, Rothery S, Krueger RR. Longterm healing of the central cornea after PRK using an excimer laser. Ophthalmology 1988;95:1411-26.

15. Binder PS. Optical problems following refractive surgery. Ophthalmology 1986;93:739-46.

16. Waring GO III, Moffitt SD, Gelender H, Laibson PR, Lindstrom RL, Myers WD, Obstbaum SA, Rowsey JJ, Safir A, Schanzlin DJ, Bourque LB (the PERK study group). Rationale for and design of the National Eye Institute Prospective Evaluation of Radial Keratotomy (PERK) study. Ophthalmology 1983;90:40-54.

17. Lin DTC, Sutton HF, Berman M. Corneal topography following excimer photorefractive keratectomy for myopia. J Cataract Refract Surg 1993;19:149-57.

18. Steinberg EB, Waring GO III. Comparison of two methods of marking the visual axis on the cornea during radial keratotomy. Am J Ophthalmol 1983; 96:605-17.

19. Lowenfeld IE, Newsome DA. Iris mechanics. I. Influence of pupil size on dynamics of pupillary movements. Am J Ophthalmol 1971;71:347-59.

20. Semmlow J. Variation in pupillomotor responsiveness with mean pupil size. Vision Res 1975;15:85-94.

21. McClusky DJ, Garbus JJ, McDonnel PJ. Optical zone shape and diurnal fluctuation after radial keratotomy. Ophthalmology 1988;95(Suppl):162-76.

22. Jones R. Do women and myopes have larger pupils? Invest Ophthalmol Vis Sci 1991;31:1413-27. 